\title{
Coagulation factors in chronic liver disease
}

\author{
G. W. K. DONAldson, S. H. DAVIES, AlEXIS DARG, AND JOHN RICHMOND
}

\author{
From the University Department of Medicine, the Department of Haematology, and \\ the Regional Blood Transfusion Service, the Royal Infirmary, Edinburgh
}

SYNOPSIS Coagulation studies were carried out on 30 patients with chronic liver disease. The clotting defect was complex and involved factors V, VII, IX (Christmas factor), and prothrombin. Some patients showed a significant depression of factor IX in the presence of a normal one-stage prothrombin time. Thrombotest was found to be a good indicator of factor IX deficiency in this group of patients and may be of use as an additional liver function test. The screening of patients with liver disease for surgery or liver biopsy should assess the coagulation factors involved in both intrinsic and extrinsic thromboplastin generation.

In 1935, Quick demonstrated a coagulation defect in liver disease when he introduced the one-stage prothrombin test (Quick, Stanley-Brown, and Bancroft, 1935). The defect has since been studied by several workers (Cowling, 1956; Rapaport, Ames, Mikkelsen, and Goodman, 1960; Hedenberg and Korsan-Bengsten, 1962; Kupfer, Gee, Ewald, and Turner, 1963) and is now known to be very complex. The finding of a marked depression of factor IX and a normal one-stage prothrombin time in one patient with cirrhosis of the liver stimulated the present detailed investigation of coagulation factors in other patients with chronic liver disease.

\section{MATERIALS AND METHODS}

Thirty patients were investigated. In most cases the diagnosis of hepatic cirrhosis was confirmed by needle biopsy of liver using the Vim-Silverman or Menghini needle or by operative liver biopsy. The main clinical details, results of routine liver function tests, and biopsy findings are given in Table I. The few patients who had received parenteral synthetic vitamin $K$ (Synkavit) previously are indicated. Peripheral blood examination was carried out using standard haematological techniques (Dacie and Lewis, 1963).

The following tests of bleeding and clotting function were undertaken: Bleeding time (Ivy, Nelson, and Bucher, 1941); clotting time in glass and siliconized tubes (Lee and White, 1913); one-stage prothrombin time using an acetone extract of human brain (Quick, 1942), the results expressed as the ratio of patient time to control time; prothrombin consumption index (as described by Dacie and Lewis, 1963); factor V activity (Quick and Stefanini, 1948); factor VII level by comparing the patient's plasma with that of a patient with congenital

Received for publication 12 July 1968. factor VII deficiency (Biggs and Macfarlane, 1962); two-stage prothrombin time (Biggs and Macfarlane, 1962); Thrombotest activity (Owren, 1959); thromboplastin generation test (Biggs and Douglas, 1953); factor IX level by a modification of the thromboplastin generation test (Biggs and Macfarlane, 1962); fibrinogen as a concentration (Biggs and Macfarlane, 1962) and as a titre using Fibrindex ${ }^{1}$ (Sharp, Howie, Biggs, and Methuen, 1958); and the euglobulin lysis time as an index of fibrinolytic activity (Biggs and Macfarlane, 1962).

\section{RESULTS}

The results of the peripheral blood counts and the various tests of bleeding and coagulation are given in Table II. Eleven patients had thrombocytopenia with platelet counts of less than $150,000 / \mathrm{c} \mathrm{mm}$; in each case this was in keeping with their degree of splenic enlargement from portal hypertension. A few subjects showed a slight prolongation of clotting time but in no case was this marked. Only two patients (nos. 16 and 17) showed a reduction in factor V activity, but the level of factor VII was found to be deficient in 10 patients. The one-stage prothrombin time was prolonged by more than three seconds in 12 parients. An abnormal prothrombin consumption index was obtained in only one patient (no. 10) who had low levels of factors VII and IX. The two-stage prothrombin time was slightly reduced in four patients (nos. 17, 18, 19, and 26).

The Thrombotest activity was considerably reduced in seven patients and was impaired in many patients. There was a serum abnormality in the thromboplastin generation test in 10 of these 
TABLE I

CLINICAL DETAILS AND RESULTS OF LIVER FUNCTION TESTS

\begin{tabular}{|c|c|c|c|c|c|c|c|c|}
\hline $\begin{array}{l}\text { Case } \\
\text { No. }\end{array}$ & Age & Sex & $\begin{array}{l}\text { Clinical } \\
\text { Diagnosis }\end{array}$ & $\begin{array}{l}\text { Biopsy } \\
\text { Confirmation }\end{array}$ & $\begin{array}{l}\text { Given } \\
\text { Vitamin } \\
\text { K }\end{array}$ & $\begin{array}{l}\text { Presenting } \\
\text { Feature }\end{array}$ & $\begin{array}{l}\text { Haematemesis }(H) \\
\text { or Melaena }(M)\end{array}$ & $\begin{array}{l}\text { History of } \\
\text { Encephalopathy }\end{array}$ \\
\hline 1 & 60 & $\mathbf{F}$ & $\begin{array}{l}\text { Portal } \\
\text { cirrhosis }\end{array}$ & Yes & Yes & Haematemesis & Repeated $\mathrm{H}$ and $\mathrm{M}$ & Yes \\
\hline 2 & 47 & $\mathbf{M}$ & $\begin{array}{l}\text { Alcoholic } \\
\text { cirrhosis }\end{array}$ & Yes & No & Anaemia & $H$ and $M$ & No \\
\hline 3 & 32 & $\mathbf{F}$ & $\begin{array}{l}\text { Portal } \\
\text { cirrhosis }\end{array}$ & Yes & No & Haematemesis & Repeated $\mathrm{H}$ and $\mathbf{M}$ & No \\
\hline 4 & 49 & $\mathbf{M}$ & $\begin{array}{l}\text { Alcoholic } \\
\text { cirrhosis }\end{array}$ & Yes & No & Anorexia & $\mathbf{M} \times 1$ & No \\
\hline 5 & 55 & $\mathbf{F}$ & $\begin{array}{l}\text { Portal } \\
\text { cirrhosis }\end{array}$ & Yes & No & Haematemesis & Repeated $\mathrm{H}$ and $\mathrm{M}$ & Yes \\
\hline 6 & 27 & $\mathbf{F}$ & $\begin{array}{l}\text { Portal } \\
\text { cirrhosis }\end{array}$ & Yes & No & Splenomegaly & - & No \\
\hline 7 & 64 & $\mathbf{F}$ & $\begin{array}{l}\text { Portal } \\
\text { cirrhosis }\end{array}$ & Yes & No & Anaemia & Repeated M & No \\
\hline 8 & 55 & $\mathbf{F}$ & $\begin{array}{l}\text { Portal } \\
\text { cirrhosis }\end{array}$ & Yes & No & Haematemesis & $\mathbf{H}$ and $\mathbf{M}$ & No \\
\hline 9 & 30 & $\mathbf{F}$ & $\begin{array}{l}\text { Portal } \\
\text { cirrhosis }\end{array}$ & Yes & Yes & Ascites & Repeated H & No \\
\hline 10 & 41 & $F$ & $\begin{array}{l}\text { Alcoholic } \\
\text { cirrhosis }\end{array}$ & Yes & No & Haematemesis & $\mathrm{H} \times 2$ & Yes \\
\hline 11 & 19 & $\mathbf{F}$ & $\begin{array}{l}\text { Portal } \\
\text { cirrhosis }\end{array}$ & Yes & No & Anaemia & Occult blood in stool & No \\
\hline 12 & 45 & $\mathbf{M}$ & $\begin{array}{l}\text { Wilson's } \\
\text { disease }\end{array}$ & $\begin{array}{l}\text { Fatty } \\
\text { degeneration }\end{array}$ & No & Tremor & - & No \\
\hline 13 & 51 & $\mathbf{M}$ & $\begin{array}{l}\text { Alcoholic } \\
\text { cirrhosis }\end{array}$ & Yes & Yes & Jaundice & Occult blood in stool & No \\
\hline 14 & 52 & $\mathbf{M}$ & $\begin{array}{l}\text { Secondary } \\
\text { carcinoma }\end{array}$ & Yes & Yes & Ascites & - & No \\
\hline 15 & 51 & $\mathbf{M}$ & $\begin{array}{l}\text { Biliary } \\
\text { cirrhosis }\end{array}$ & Yes & Yes & Pruritus & - & No \\
\hline 16 & 71 & $\mathbf{F}$ & $\begin{array}{l}\text { Portal } \\
\text { cirrhosis }\end{array}$ & Yes & No & Splenomegaly & - & Yes \\
\hline 17 & 42 & $\mathbf{M}$ & $\begin{array}{l}\text { Portal } \\
\text { cirrhosis }\end{array}$ & Yes & No & Melaena & Repeated $H$ and $M$ & Yes \\
\hline 18 & 46 & $\mathbf{M}$ & $\begin{array}{l}\text { Portal } \\
\text { cirrhosis }\end{array}$ & Yes & No & Haematemesis & Repeated $H$ and $M$ & No \\
\hline 19 & 22 & $\mathbf{F}$ & $\begin{array}{l}\text { Portal } \\
\text { cirrhosis }\end{array}$ & Yes & No & Malaise & $H$ and $M$ & No \\
\hline 20 & 60 & $\mathbf{M}$ & $\begin{array}{l}\text { Portal } \\
\text { cirrhosis }\end{array}$ & Yes & No & Jaundice & - & No \\
\hline 21 & 67 & $\mathbf{M}$ & $\begin{array}{l}\text { Alcoholic } \\
\text { cirrhosis }\end{array}$ & Yes & No & Jaundice & - & Yes \\
\hline 22 & 37 & $\mathbf{M}$ & $\begin{array}{l}\text { Portal } \\
\text { cirrhosis }\end{array}$ & Yes & No & Jaundice & - & No \\
\hline 23 & 37 & $\mathbf{F}$ & $\begin{array}{l}\text { Alcoholic } \\
\text { cirrhosis }\end{array}$ & Yes & No & Malaise & - & No \\
\hline 24 & 54 & $\mathbf{M}$ & $\begin{array}{l}\text { Alcoholic } \\
\text { cirrhosis }\end{array}$ & No & Yes & Jaundice & - & No \\
\hline 25 & 63 & $\mathbf{F}$ & $\begin{array}{l}\text { Alcoholic } \\
\text { cirrhosis }\end{array}$ & No & No & Haematemesis & Repeated M & No \\
\hline 26 & 66 & $\mathbf{F}$ & $\begin{array}{l}\text { Portal } \\
\text { cirrhosis }\end{array}$ & Yes & No & Jaundice & $\mathbf{M} \times 1$ & Yes \\
\hline 27 & 61 & $\mathbf{M}$ & $\begin{array}{l}\text { Portal } \\
\text { cirrhosis }\end{array}$ & Yes & No & Oedema & $\mathbf{M} \times \mathbf{2}$ & Yes \\
\hline 28 & 62 & $\mathbf{F}$ & $\begin{array}{l}\text { Portal } \\
\text { cirrhosis }\end{array}$ & No & No & $\begin{array}{l}\text { Subarachnoid } \\
\text { haemorrhage }\end{array}$ & - & No \\
\hline 29 & 70 & $\mathbf{M}$ & $\begin{array}{l}\text { Portal } \\
\text { cirrhosis }\end{array}$ & Yes & No & Angina & - & No \\
\hline 30 & 51 & $F$ & $\begin{array}{l}\text { Portal } \\
\text { cirrhosis }\end{array}$ & Yes & No & Haematemesis & Repeated $\mathbf{H}$ and $M$ & Yes \\
\hline
\end{tabular}


TABLE I-continued

CLINICAL DETAILS AND RESULTS OF LIVER FUNCTION TESTS

\begin{tabular}{|c|c|c|c|c|c|c|c|c|c|c|}
\hline \multirow[t]{2}{*}{ Splenomegaly } & \multirow{2}{*}{$\begin{array}{l}\text { Serum } \\
\text { Bilirubin } \\
(m g \%)\end{array}$} & \multirow{2}{*}{$\begin{array}{l}\text { Serum Alkaline } \\
\text { Phosphatase } \\
\text { (King-Armstrong units) }\end{array}$} & \multirow{2}{*}{$\begin{array}{l}\text { Serum } \\
\text { Thymol } \\
\text { Turbidity }\end{array}$} & \multirow{2}{*}{$\begin{array}{l}\text { Serum } \\
\text { Transaminase } \\
\text { SGPT }\end{array}$} & \multirow{2}{*}{$\begin{array}{l}\text { Total } \\
\text { Serum } \\
\text { Proteins } \\
(\mathrm{g} \%)\end{array}$} & \multirow{2}{*}{$\begin{array}{l}\text { Albumin } \\
\text { (\% of total) }\end{array}$} & \multicolumn{4}{|c|}{ Globulin ( $\%$ of total) } \\
\hline & & & & & & & $a_{1}$ & $a_{2}$ & $\beta$ & $\boldsymbol{\gamma}$ \\
\hline 2 f.b. & $3 \cdot 6$ & 36 & 7 & $<20$ & $6 \cdot 5$ & $45^{3 \cdot 0}$ & $5^{0.3}$ & $11^{0.7}$ & $8^{0.5}$ & $30^{2 \cdot 0}$ \\
\hline 1 f.b. & $1 \cdot 6$ & 12 & 5 & $<\mathbf{2 0}$ & $6 \cdot 5$ & 42 & $\begin{array}{l}0 \cdot 1 \\
2\end{array}$ & $\begin{array}{l}0 \cdot 6 \\
9\end{array}$ & $\begin{array}{l}1 \cdot 1 \\
17\end{array}$ & $\begin{array}{l}2 \cdot 0 \\
30\end{array}$ \\
\hline Splenectomy & $1 \cdot 7$ & 18 & 6 & $<20$ & $8 \cdot 1$ & $\begin{array}{l}3 \cdot 3 \\
41\end{array}$ & $\begin{array}{l}0 \cdot 2 \\
2\end{array}$ & $\begin{array}{l}0 \cdot 6 \\
8\end{array}$ & $15^{1 \cdot 2}$ & $\begin{array}{l}2 \cdot 8 \\
34\end{array}$ \\
\hline Tip & 0.6 & 21 & 1 & 43 & $7 \cdot 6$ & $\begin{array}{c}4 \cdot 0 \\
52\end{array}$ & $\begin{array}{c}0 \cdot 3 \\
4\end{array}$ & $\begin{array}{l}0.8 \\
11\end{array}$ & $\begin{array}{c}0.8 \\
11\end{array}$ & $2^{1 \cdot 7}$ \\
\hline Splenectomy & $0 \cdot 8$ & 7 & 1 & $<20$ & $6 \cdot 8$ & $\begin{array}{l}3 \cdot 3 \\
48\end{array}$ & $\begin{array}{l}0 \cdot 3 \\
4\end{array}$ & $\begin{array}{l}0 \cdot 8 \\
12\end{array}$ & $\begin{array}{l}0 \cdot 8 \\
12\end{array}$ & ${ }_{24}^{1 \cdot 6}$ \\
\hline 5 f.b. & 0.9 & 6 & 1 & $<20$ & $6 \cdot 1$ & $\begin{array}{l}4 \cdot 1 \\
67\end{array}$ & $\begin{array}{l}0 \cdot 1 \\
2\end{array}$ & $\begin{array}{l}0.5 \\
9\end{array}$ & $\begin{array}{l}0.7 \\
11\end{array}$ & $\begin{array}{l}0.7 \\
11\end{array}$ \\
\hline - & $1 \cdot 7$ & 30 & 7 & 27 & $5 \cdot 7$ & $2 \cdot 2 \cdot 5$ & $\begin{array}{l}0 \cdot 3 \\
6\end{array}$ & $\begin{array}{c}0 \cdot 6 \\
10\end{array}$ & $\begin{array}{l}0 \cdot 7 \\
12\end{array}$ & 28 \\
\hline 2 f.b. & 0.6 & 19 & 6 & $<\mathbf{2 0}$ & $6 \cdot 3$ & $2 \cdot 2 \cdot 2$ & $\begin{array}{l}0 \cdot 3 \\
5\end{array}$ & $\begin{array}{l}0 \cdot 9 \\
14\end{array}$ & $21^{1 \cdot 3}$ & 25 \\
\hline 3 f.b. & $2 \cdot 2$ & 45 & 3 & 30 & 6.9 & $56^{3 \cdot 8}$ & $\begin{array}{l}0 \cdot 3 \\
4\end{array}$ & $11^{0 \cdot 8}$ & $11^{0 \cdot 8}$ & $18^{1 \cdot 2}$ \\
\hline Tip & 0.5 & 40 & 8 & 20 & $7 \cdot 1$ & $\begin{array}{l}3 \cdot 1 \\
43\end{array}$ & $\begin{array}{l}0 \cdot 3 \\
4\end{array}$ & $\begin{array}{l}0 \cdot 5 \\
7\end{array}$ & $\begin{array}{l}0 \cdot 6 \\
8\end{array}$ & $\begin{array}{l}2 \cdot 7 \\
38\end{array}$ \\
\hline- & $0 \cdot 3$ & 6 & 1 & $<20$ & $7 \cdot 7$ & $\begin{array}{l}3 \cdot 8 \\
49\end{array}$ & $\begin{array}{l}0 \cdot 5 \\
6\end{array}$ & $\begin{array}{l}0.9 \\
12\end{array}$ & $13^{1 \cdot 0}$ & $20^{1 \cdot 5}$ \\
\hline- & 0.7 & 8 & 3 & $<20$ & $7 \cdot 6$ & $50^{3 \cdot 8}$ & $\begin{array}{l}0 \cdot 3 \\
4\end{array}$ & 14 & $\begin{array}{l}0.8 \\
11\end{array}$ & $21 \cdot 6$ \\
\hline- & $14 \cdot 6$ & 11 & 3 & 62 & 6.8 & $40^{2 \cdot 7}$ & $\begin{array}{c}0 \cdot 7 \\
10\end{array}$ & $17^{1 \cdot 2}$ & $14^{1 \cdot 0}$ & $19^{1 \cdot 3}$ \\
\hline 3 f.b. & $2 \cdot 4$ & 20 & 1 & 15 & $5 \cdot 4$ & $\begin{array}{c}2 \cdot 8 \\
51\end{array}$ & $\begin{array}{l}0.4 \\
8\end{array}$ & $\begin{array}{c}0.9 \\
17\end{array}$ & $\begin{array}{c}0.6 \\
11\end{array}$ & $\begin{array}{l}0 \cdot 7 \\
13\end{array}$ \\
\hline- & $17 \cdot 5$ & 95 & 3 & 68 & $6 \cdot 5$ & $\begin{array}{l}2 \cdot 7 \\
41\end{array}$ & $\begin{array}{l}0 \cdot 5 \\
7\end{array}$ & 30 & $17^{1 \cdot 1}$ & $\begin{array}{l}0 \cdot 3 \\
5\end{array}$ \\
\hline 2 f.b. & $1 \cdot 2$ & 20 & 3 & 25 & $6 \cdot 2$ & $5^{3 \cdot 6}$ & $\begin{array}{c}0.6 \\
10\end{array}$ & $\begin{array}{c}0.8 \\
13\end{array}$ & $\begin{array}{l}0 \cdot 4 \\
6\end{array}$ & $\begin{array}{c}0.8 \\
13\end{array}$ \\
\hline- & $4 \cdot 0$ & 9 & $>10$ & 43 & $6 \cdot 8$ & $40^{2 \cdot 7}$ & $\begin{array}{l}0 \cdot 1 \\
2\end{array}$ & $\begin{array}{l}0 \cdot 6 \\
9\end{array}$ & $17^{1 \cdot 2}$ & ${ }^{2 \cdot 2}$ \\
\hline Splenectomy & 0.4 & 16 & 2 & $<20$ & $7 \cdot 0$ & $5^{3 \cdot 9}$ & $\begin{array}{l}0 \cdot 1 \\
2\end{array}$ & $\begin{array}{c}0 \cdot 8 \\
12\end{array}$ & $\begin{array}{l}0.9 \\
13\end{array}$ & $18^{1 \cdot 3}$ \\
\hline- & $1 \cdot 7$ & 3 & 1 & $<20$ & 6.9 & $\begin{array}{l}4 \cdot 2 \\
61\end{array}$ & $\begin{array}{l}0 \cdot 3 \\
4\end{array}$ & $\begin{array}{c}0 \cdot 8 \\
12\end{array}$ & $\begin{array}{l}0.9 \\
13\end{array}$ & $\begin{array}{l}0 \cdot 7 \\
10\end{array}$ \\
\hline- & 0.6 & 30 & & 75 & $6 \cdot 2$ & $\begin{array}{l}2 \cdot 9 \\
46\end{array}$ & $\begin{array}{l}0 \cdot 3 \\
4\end{array}$ & $\begin{array}{c}0 \cdot 7 \\
11\end{array}$ & $\begin{array}{c}0.9 \\
14\end{array}$ & $2^{1 \cdot 6}$ \\
\hline- & 0.6 & 12 & 2 & $<20$ & $6 \cdot 5$ & $\begin{array}{l}3.6 \\
56\end{array}$ & $\begin{array}{l}0.3 \\
4\end{array}$ & $\begin{array}{c}0 \cdot 7 \\
11\end{array}$ & $\begin{array}{c}0.7 \\
10\end{array}$ & $19^{1 \cdot 2}$ \\
\hline - & $2 \cdot 5$ & 27 & 6 & 25 & $5 \cdot 2$ & $\begin{array}{c}2 \cdot 1 \\
41\end{array}$ & $\begin{array}{l}0 \cdot 4 \\
8\end{array}$ & $\begin{array}{l}0.6 \\
12\end{array}$ & $\begin{array}{c}0.5 \\
10\end{array}$ & 29 \\
\hline- & 0.2 & 9 & 2 & $<20$ & $6 \cdot 1$ & $6^{3 \cdot 8}$ & $\begin{array}{l}0 \cdot 1 \\
2\end{array}$ & $\begin{array}{l}0.5 \\
9\end{array}$ & $11^{0.7}$ & $\begin{array}{c}0.9 \\
15\end{array}$ \\
\hline- & $1 \cdot 2$ & 9 & 5 & 25 & $6 \cdot 8$ & $37^{2 \cdot 5}$ & $\begin{array}{l}0 \cdot 5 \\
7\end{array}$ & $\begin{array}{l}0 \cdot 8 \\
12\end{array}$ & $0^{0.8}$ & 33 \\
\hline 3 f.b. & 0.5 & 5 & 1 & $<20$ & $7 \cdot 1$ & $6^{4 \cdot 5}$ & $\begin{array}{l}0 \cdot 2 \\
3\end{array}$ & $\begin{array}{l}0.9 \\
13\end{array}$ & $\begin{array}{c}0.8 \\
11\end{array}$ & $\begin{array}{l}0 \cdot 6 \\
9\end{array}$ \\
\hline- & 0.8 & 7 & 4 & $<20$ & 6.4 & $4^{3 \cdot 1}$ & $\begin{array}{l}0 \cdot 1 \\
2\end{array}$ & $\begin{array}{l}0 \cdot 4 \\
6\end{array}$ & $\begin{array}{l}0.8 \\
13\end{array}$ & $\begin{array}{l}2 \cdot 0 \\
31\end{array}$ \\
\hline- & $5 \cdot 8$ & 40 & 8 & 40 & 6.0 & $\begin{array}{c}2 \cdot 9 \\
48\end{array}$ & $\begin{array}{l}0 \cdot 2 \\
3\end{array}$ & $\begin{array}{l}0.9 \\
15\end{array}$ & $\begin{array}{l}0 \cdot 7 \\
12\end{array}$ & $22^{1 \cdot 3}$ \\
\hline 2 f.b. & $1 \cdot 1$ & 14 & 6 & $<20$ & $6 \cdot 8$ & $40^{2 \cdot 7}$ & $\begin{array}{l}0 \cdot 2 \\
3\end{array}$ & $\begin{array}{l}0.8 \\
11\end{array}$ & $18^{1 \cdot 2}$ & $2^{1 \cdot 9}$ \\
\hline- & 0.4 & 31 & 3 & 29 & $8 \cdot 1$ & $\begin{array}{c}3 \cdot 1 \\
39\end{array}$ & $\begin{array}{l}0 \cdot 2 \\
2\end{array}$ & $16^{1 \cdot 3}$ & $17^{1 \cdot 4}$ & 26 \\
\hline 4 f.b. & $1 \cdot 3$ & 50 & & 52 & $7 \cdot 2$ & $3^{2 \cdot 5}$ & $\begin{array}{l}0 \cdot 2 \\
3\end{array}$ & $\begin{array}{l}0 \cdot 7 \\
10\end{array}$ & $14^{1 \cdot 0}$ & $\begin{array}{l}2 \cdot 8 \\
39\end{array}$ \\
\hline
\end{tabular}


TABLE II

PERIPHERAL BLOOD FINDINGS AND RESULTS OF TESTS OF BLEEDING AND CLOTTING FUNCTIONS

\begin{tabular}{|c|c|c|c|c|c|c|c|c|c|}
\hline \multirow[t]{2}{*}{ Case No. } & \multirow{2}{*}{$\begin{array}{l}\mathrm{Hb}(\mathrm{g} / 100 \mathrm{ml}) \\
(100 \% \text { value }= \\
14.6 \mathrm{~g} / 100 \mathrm{ml})\end{array}$} & \multirow{2}{*}{$\begin{array}{l}W B C \\
\text { per c mm }\end{array}$} & \multirow{2}{*}{$\begin{array}{l}\text { Platelets } \\
\text { per c } \mathrm{mm}\end{array}$} & \multirow{2}{*}{$\begin{array}{l}\text { Hess } \\
\text { Test }\end{array}$} & \multirow{2}{*}{$\begin{array}{l}\text { Bleeding } \\
\text { Time } \\
\text { (Ivy) }\end{array}$} & \multicolumn{2}{|c|}{ Clotting Time } & \multirow{2}{*}{$\begin{array}{l}\text { Prothrombin } \\
\text { Ratio to } \\
\text { Normal }\end{array}$} & \multirow{2}{*}{$\begin{array}{l}\text { Prothrombin } \\
\text { Consumption } \\
\text { Index }\end{array}$} \\
\hline & & & & & & Glass & Silicone & & \\
\hline
\end{tabular}

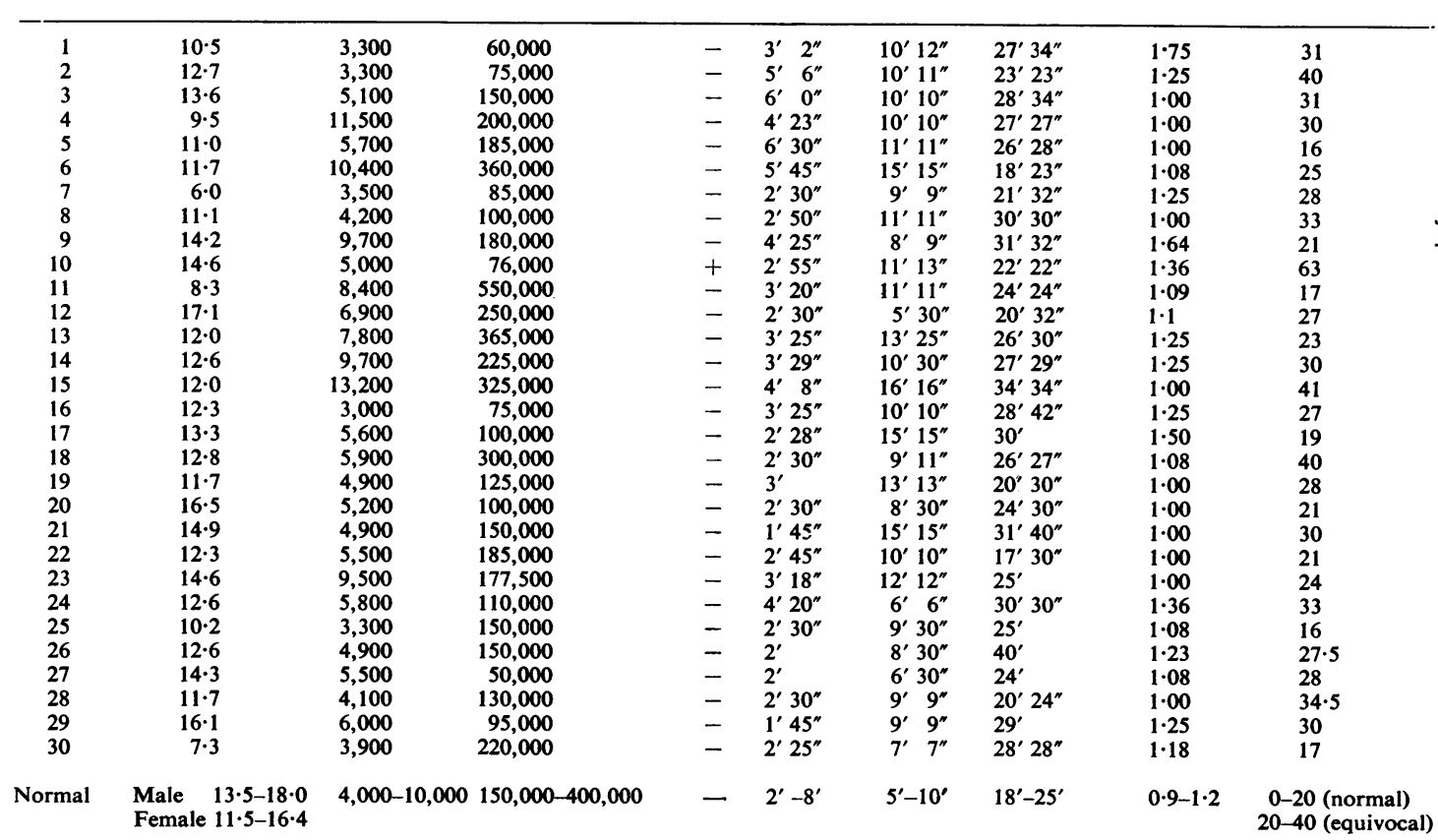

patients; this was corrected by normal serum but not by serum from a patient with Christmas disease. Factor IX was low in all of these 10 patients with abnormal thromboplastin generation and was also moderately reduced in two others.

In all patients the fibrinogen titre and concentration were normal. Patient no. 3 showed a euglobulin lysis time less than normal but as judged by this method no patient had evidence of gross fibrinolysis.

There was significant correlation between the assay of factor VII and the one-stage prothrombin time $(r=0.76 ; P<0.001 ; n=30)$. There was no significant correlation between the results of factor IX assay and the one-stage prothrombin time $(r=0.39 ; 0.05>p>0.01 ; n=30)$. Good correlation existed between the results of the Thrombotest and the assay of factor IX $(r=0.78 ; \mathrm{P}<0.001$; $\mathrm{n}=30$ ) (Fig. 1), and fair correlation between Thrombotest activity and factor VII level $(r=0.55$; $0.01>\mathrm{P}>0.001 ; \mathrm{n}=30$ ).

\section{DISCUSSION}

The defect in coagulation in patients with chronic hepatocellular disease is rarely due to a single abnormality. Like others (Kupfer et al, 1963) we have found the clotting and bleeding times to be of little value. We have confirmed that reduction of factors V, VII, IX, and prothrombin may exist.

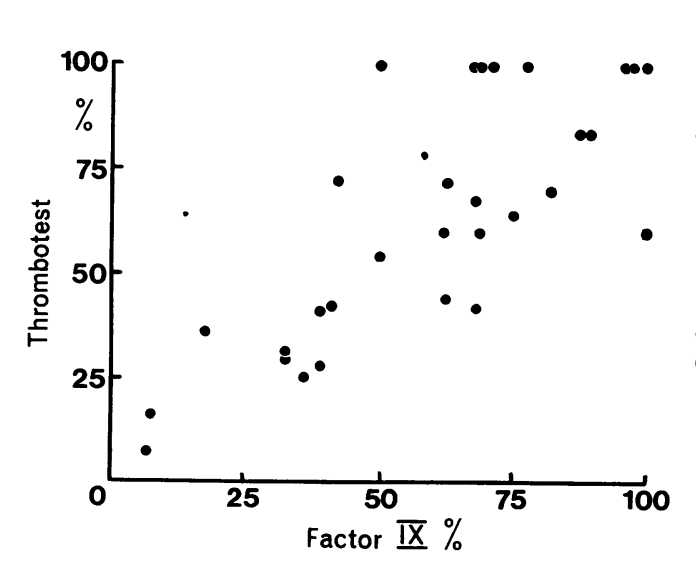

FIG. 1. Correlation between results of the Thrombotest and the assay of factor $I X$. 
TABLE II-continued

PERIPHERAL BLOOD FINDINGS AND RESULTS OF TESTS OF BLEEDING AND CLOTTING FUNCTIONS

\begin{tabular}{|c|c|c|c|c|c|c|c|c|c|c|}
\hline \multirow{3}{*}{$\begin{array}{l}\text { Factor } \\
V \\
(\%)\end{array}$} & \multirow{3}{*}{$\begin{array}{l}\text { Factor } \\
\text { VII } \\
(\%)\end{array}$} & \multirow{3}{*}{$\begin{array}{l}\text { Two-stage } \\
\text { Prothrombin } \\
(\%)\end{array}$} & \multicolumn{2}{|c|}{ Thrombotest $(\%)$} & \multirow{3}{*}{$\begin{array}{l}\text { Thromboplastin } \\
\text { Generation Test }\end{array}$} & \multirow{3}{*}{$\begin{array}{l}\text { Factor } \\
I X \\
(\%)\end{array}$} & \multicolumn{3}{|l|}{ Fibrinogen } & \multirow{3}{*}{$\begin{array}{l}\text { Euglobulin } \\
\text { Lysis Time } \\
\text { (min) }\end{array}$} \\
\hline & & & Control & Patient & & & Concentration & Thrombi & Titre & \\
\hline & & & & & & & & Control & Patient & \\
\hline
\end{tabular}

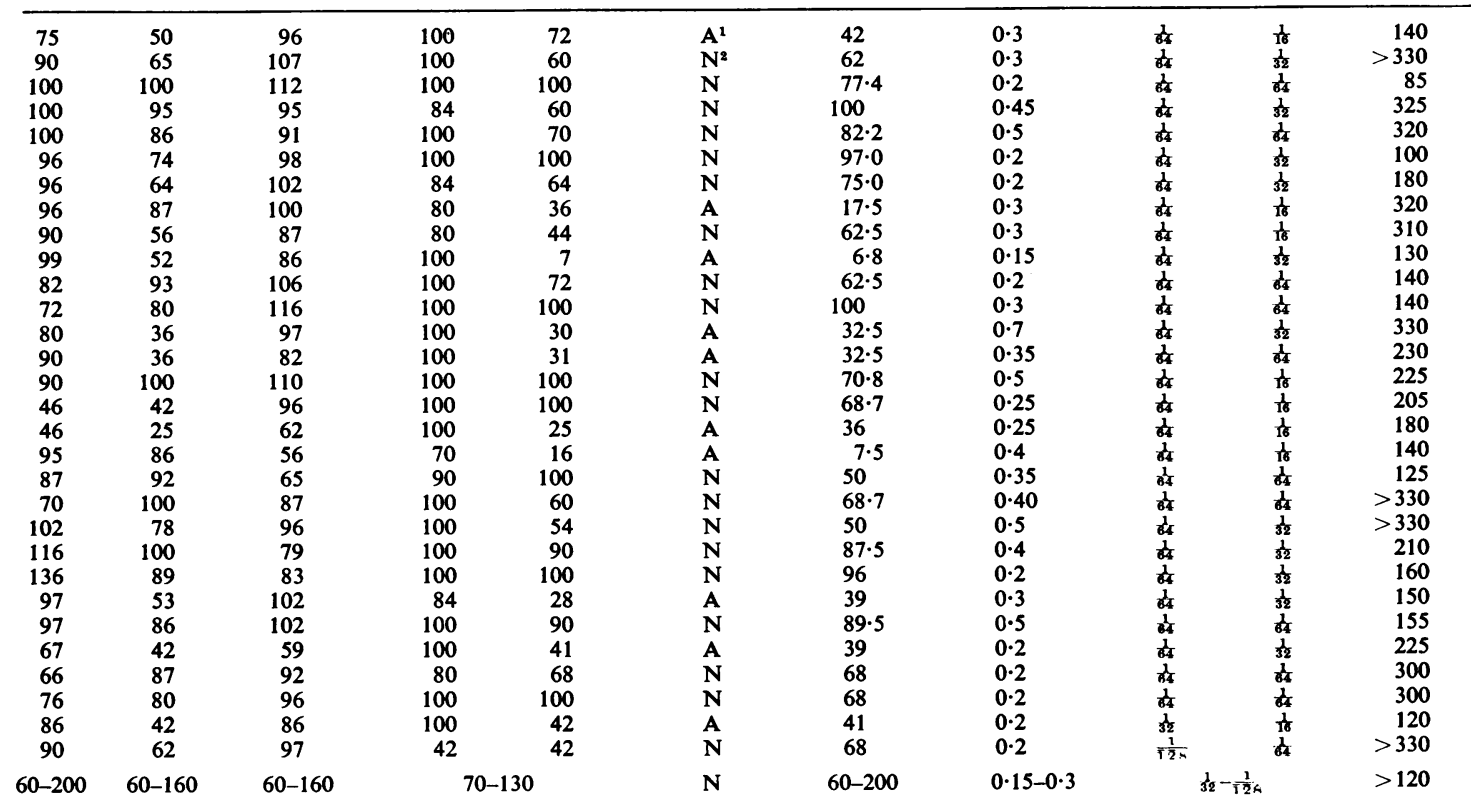

${ }^{\mathbf{1}} \mathbf{A}=$ abnormal.

${ }^{2} \mathbf{N}=$ normal

Cowling (1956) stated that a prolonged one-stage prothrombin time is caused mainly by combined deficiencies of factors V and VII. In his patients and in those of Finkbiner, McGovern, Goldstein, and Bunker (1959) lack of factor IX was found only in patients with prolonged prothrombin times. Our results indicate that prolongation of the one-stage prothrombin time correlates well with lack of factor VII but that factor IX deficiency, which was present in many patients, is not always accompanied by a prolonged one-stage prothrombin time. On the other hand we have found Thrombotest to be a good indicator of deficiency of factor IX (Fig. 1) in this group of patients, although Thrombotest has been reported as relatively insensitive in detecting deficiency of factor IX in the plasma of patients with Christmas disease or in patients who are receiving therapy with phenindione (Denson, 1961). We do not have an explanation for this (unexpected) finding, although we note that the occasional patient with congenital factor IX deficiency may have a prolonged Thrombotest (Kidd, Denson, and Biggs, 1963). Some of our patients showed depression of factor IX despite normal routine liver function tests and normal serum albumin levels. The one-stage prothrombin time is said to be a useful liver function test (Williams, 1965), but Thrombotest might be preferable as it may detect abnormalities of intrinsic as well as of extrinsic thromboplastin generation, both of which are affected by liver disease. Disorders of haemostatic function are extremely common in patients with liver disease (Hedenberg and KorsanBengsten, 1962) and Thrombotest may be of diagnostic use in the assessment of such patients as suggested originally by Owren (1959).

Haemorrhage in patients with liver disease may occur in the presence of apparently normal coagulation. Most authors consider that a clotting defect contributes to the severity of the bleeding although it may not precipitate it (Cowling, 1956; Finkbiner et al, 1959; Kupfer et al, 1963). It was not possible from a study of our patients to assess the relative importance of the coagulation defect in the causation of bleeding. In none of the patients studied was there abnormal bleeding after needle biopsy of the liver. Further, although some of our patients showed marked abnormalities of coagulation, there was no evidence in this group that such abnormalities 
affected the prognosis. It is difficult to evaluate the significance of a single clotting defect, and even more difficult to assess minor deficiencies in several coagulation factors. Moreover the relative importance of defective coagulation, abnormal fibrinolysis, thrombocytopenia, portal hypertension, and the presence of oesophageal varices in precipitating or prolonging haemorrhage has still to be established.

It is commonly recommended (Sherlock, 1963) that the one-stage prothrombin time be estimated as a screening precaution before needle biopsy of the liver is undertaken. However, since lack of factor IX is not shown by this test, we suggest that in the meantime patients with liver disease in whom bleeding and clotting functions need to be assessed, eg, for liver biopsy, should be investigaged with a test to measure intrinsic thromboplastin generation, as well as with the more customary one-stage prothrombin time, which depends on extrinsic thromboplastin generation and the prothrombin content of the blood. We regard such a screen as a minimum and, in practice, we perform the following investigations for such patients: peripheral blood counts including platelet count; bleeding time; whole blood clotting time; one-stage prothrombin time; Thrombotest or partial thromboplastin time
(Matchett and Ingram, 1965); prothrombin consumption index; and euglobulin lysis time.

\section{REFERENCES}

Biggs, R., and Douglas, A. S. (1953). J. clin. Path., 6, 23. , and Macfarlane, R. G. (1962). Human Blood Coagulation and Its Disorders, 3rd ed. Blackwell Scientific Publications, Oxford. Cowling, D. C. (1956). J. clin. Path., 9, 347.

Dacie, J. V., and Lewis, S. M. (1963). Practical Haematology, 2nd ed.œ̂ Churchill, London.

Denson, K. W. (1961). Brit. med. J., 1, 1205.

Finkbiner, R. B., McGovern, J. J., Goldstein, R., and Bunker, J. P.(1959). Amer. J. Med., 26, 199.

Hedenberg, L., and Korsan-Bengsten, K. (1962). Acta med. scand. 172, 229.

Ivy, A. C., Nelson, D., and Bucher, G. R. (1941). J. Lab. clin. Med.융 26, 1812.

Kidd, P., Denson, K. W. E., and Biggs, R. (1963). Lancet, 2, 522.

Kupfer, H. G., Gee, W., Ewald, A. T., and Turner, M. E. (1963) N Thrombos. Diathes. haemorrh. (Stuttg.), 10, 317.

Lee, R. I., and White, P. D. (1913). Amer. J. med. Sci., 145, 495.

Matchett, M. O., and Ingram, G. I. C. (1965). J. clin. Path., 18, 465 .

Owren, P. A. (1959). Lancet, 2, 754.

Quick, A. J. (1942). The Haemorrhagic Diseases and the Physiology of Haemostasis. Thomas, Springfield, III.

- Stanley-Brown, M., and Bancroft, F. W. (1935). Amer. J. med Sci., 190, 501.

- and Stefanini, M. (1948). J. Lab. clin. Med., 33, 819.

Rapaport, S. I., Ames, S. B., Mikkelsen, S., and Goodman, J. R. (1960). New Engl. J. Med., 263, 278.

Sharp, A. A., Howie, B., Biggs, R., and Methuen, D. T. (1958)ฤ Lancet, 2, 1309.

Sherlock, S. (1963). Diseases of the Liver and Biliary System, 3rd ed.D Blackwell Scientific Publications, Oxford.

Williams, R. (1965). Anaesthesia, 20, 3. 